\title{
Optimal Testing for Diagnosis of Fructose Malabsorption: Under-dosage Leads to False Negative Intolerance Test
}

TO THE EDITOR: With great interest we have been following the discussion about our study published in Journal of Neurogastroenterology and Motility. ${ }^{1}$ We highly estimate the recent constructive comments expressed by the authors Erdogan and colleagues $^{2}$ and would like to make some conclusive remarks.

We fully agree with the authors that all patients with irritable bowel syndrome (IBS)-like symptoms should undergo breath testing and have specifically stated this in our manuscript. Thus, we are not sure where Erdogan et $\mathrm{al}^{2}$ got the notion that such tests should only be performed for research purposes.

We furthermore agree with Erdogan et $\mathrm{al}^{2}$ that 2 consecutive breath tests are impractical, especially considering the bothersome symptoms that patients experience during and after testing. Therefore, we favor the breath test with $50 \mathrm{~g}$ fructose. From our yet unpublished data we know that $\mathrm{H}_{2}$ breath tests with $50 \mathrm{~g}$ fructose identify patients with IBS-like symptoms who will benefit from a fructose-reduced diet. If only $25 \mathrm{~g}$ tests were performed, an important subgroup (the ones with symptomatic $50 \mathrm{~g}$ breath test) would be missed although they greatly benefit from a detailed dietary counseling, while patients with positive $25 \mathrm{~g}$ breath test already benefit from brief dietary advice as shown by a decline in gastrointestinal (GI) symptoms, improved quality of life and state of health. In patients with severe fructose malabsorption only the detailed dietary advice regimen resulted in improvement of GI symptoms and perceived state of health. This is our rationale to perform a breath test with $50 \mathrm{~g}$ of fructose.

The German consensus paper on clinically relevant breath tests in gastroenterological diagnostics does not always recommend a test with $50 \mathrm{~g}$. It points out that in case of highly suspected fructose malabsorption, the high dose test should be performed first and in case of a positive outcome, an additional $25 \mathrm{~g}$ test enhances specificity. ${ }^{3}$

On the other hand, the rationale to start with the low dose is that healthy volunteers almost never malabsorb $25 \mathrm{~g}$. Thus, the test has an excellent specificity because, according to test criteria, there are rarely false positives. However, we feel that the low dose test lacks sensitivity because a proportion of negative low dose breath testers will still respond to a fructose elimination diet.

To deal with the lower specificity in the high dose test, observing symptoms during the test can help: increased $\mathrm{H}_{2}$ excretion combined with typical symptoms triggered by the high dose challenge is a good criterion to distinguish between true and false positive patients, and healthy volunteers barely report symptoms even though they may have elevated $\mathrm{H}_{2}$ excretion. ${ }^{4}$ Some healthy subjects and patients with IBS-like symptoms are able to fully absorb $50 \mathrm{~g}$ of fructose because otherwise, every test person would have significant $\mathrm{H}_{2}$ excretion and symptoms. In our study $64 \%$ were symptomatic fructose malabsorbers with a $50 \mathrm{~g}$ test dose, which in reverse implies that $36 \%$ of patients with unclear abdominal discomfort were able to absorb $50 \mathrm{~g}$ of fructose without clinical symptoms.

We disagree with the authors that amounts $>25 \mathrm{~g}$ of fructose are nowadays not consumed at once. As recently outlined, a 16-oz bottle of apple juice may contain $>30 \mathrm{~g}$ fructose and a 22-oz soft drink could contain approximately $30 \mathrm{~g}$ to $40 \mathrm{~g}$ depending on the percent fructose in the corn syrup sweetener, both representing liquids regularly consumed by a large proportion of the population.

As clinicians we will accept false positives. They may undergo a 4-6 weeks dietary trial and not respond. But it is unpleasant to exclude patients, in whom most of the treatments have failed, from a fructose elimination diet that works most of the time in these patients because their diagnosis was missed. Thus, we find it more appropriate to use the high dose test with $50 \mathrm{~g}$ because we feel that it is clinically more relevant. 
Miriam Goebel-Stengel and Hubert Mönnikes Department of Internal Medicine, Institute of Neurogastroenterology and Motility, Martin-Luther Hospital, Academic Teaching Institution of Charité - University Medical Center, Berlin, Germany

1. Goebel-Stengel M, Stengel A, Schmidtmann M, Voort I, Kobelt P, Mönnikes H. Unclear abdominal discomfort: pivotal role of carbohydrate malabsorption. J Neurogastroenterol Motil 2014;20:228235.

2. Erdogan A, Adame EC, Yu S, Rattanakovit K, Rao SS. Optimal testing for diagnosis of fructose intolerance: over-dosage leads to false positive intolerance test. J Neurogastroenterol Motil 2014;20: 560.

3. Keller J, Franke A, Storr M, et al. [Clinically relevant breath tests in gastroenterological diagnostics--recommendations of the German Society for Neurogastroenterology and Motility as well as the German Society for Digestive and Metabolic Diseases.] Z
Gastroenterol 2005;43:1071-1090. [German]

4. Murray K, Wilkinson-Smith V, Hoad C, et al. Differential effects of FODMAPs (fermentable oligo-, di-, mono-saccharides and polyols) on small and large intestinal contents in healthy subjects shown by MRI. Am J Gastroenterol 2014;109:110-119.

5. Beyer PL, Caviar EM, McCallum RW. Fructose intake at current levels in the United States may cause gastrointestinal distress in normal adults. J Am Diet Assoc 2005;105:1559-1566.

6. Matthews RH, Pehrsson PR, Farhat-Sabat M. Sugar content of selected foods: Individual and total sugars. Publication No. HER 48. Washington: US Department of Agriculture, Human Nutrition Information Service 1987.

\section{Conflicts of interest: None.}

